\title{
Structures and coalescence behavior of size-selected silicon nanoclusters studied by surface-plasmon-polariton enhanced Raman spectroscopy
}

\author{
E. C. Honea \\ Lawrence Livermore National Laboratories, Livermore, California 94550
}

A. Ogura

NEC, Silicon Systems Research Laboratories, Tsukuba, Japan

D. R. Peale, C. Félix, ${ }^{\text {a) }}$ C. A. Murray, K. Raghavachari, and W. O. Sprenger

Lucent Technologies, Bell Laboratories, Murray Hill, New Jersey 07974-0636

M. F. Jarrold

Department of Chemistry, Northwestern University, Evanston, Illinois 60208

W. L. Brown

Lucent Technologies, Bell Laboratories, Murray Hill, New Jersey 07974-0636

(Received 14 October 1998; accepted 5 March 1999)

\begin{abstract}
The structures and coalescence behavior of size-selected, matrix-isolated silicon clusters have been studied using surface-plasmon-polariton (SPP) enhanced Raman spectroscopy. The cluster ions were produced in a laser vaporization source, mass selected then deposited into a co-condensed matrix of $\mathrm{Ar}, \mathrm{Kr}$ or $\mathrm{N}_{2}$ on a liquid $\mathrm{He}$ cooled substrate. Raman spectra from monodisperse samples of the smaller clusters studied, $\mathrm{Si}_{4}, \mathrm{Si}_{6}$ and $\mathrm{Si}_{7}$, show sharp, well-resolved, vibrations which are in good agreement with predictions based on ab initio calculations. From these comparisons we confirm that $\mathrm{Si}_{4}$ is a planar rhombus, and assign $\mathrm{Si}_{6}$ as a distorted octahedron and $\mathrm{Si}_{7}$ as a pentagonal bypyramid. $\mathrm{Si}_{5}$ depositions down to $5 \mathrm{eV}$ did not reveal a measurable Raman spectrum under our experimental conditions. Evidence for cluster-cluster aggregation (or fragmentation) was observed under some conditions, even for a "magic number" cluster such as $\mathrm{Si}_{6}$. The spectra of the aggregated small clusters were identical to those observed for directly deposited larger cluster "bands," such as $\mathrm{Si}_{25-35}$. The Raman spectra of the aggregated clusters bear some similarity to those of bulk amorphous silicon. Cluster-deposited thin films were prepared by sublimating the matrix material. Even under these "soft landing" conditions, changes in the Raman spectrum are observed with the thin films showing even greater similarity to amorphous silicon. (C) 1999 American Institute of Physics. [S0021-9606(99)70521-0]
\end{abstract}

\section{INTRODUCTION}

In recent years, metal and semiconductor clusters made up of 2-100 atoms have been the topics of intense activity and interest. ${ }^{1}$ Silicon clusters, in particular, have demonstrated many fascinating properties. ${ }^{2-4}$ While impressive progress has been made in the understanding of this range of matter between atoms and bulk materials, structural information has been more difficult to obtain. High level calculations ${ }^{5-7}$ and studies using empirical potentials ${ }^{8,9}$ predict that silicon clusters differ significantly from the bulk crystal lattice and surfaces, i.e., they are extensively reconstructed compared to microcrystal fragments. Up to this point, experimental information about the structures was either indirect ${ }^{10}$ or limited to very small clusters of up to four atoms. ${ }^{11-13}$ This lack of knowledge about structures has limited our understanding of the physical, electronic and chemical properties of these clusters.

Many of the observed properties of silicon clusters have been interpreted as evidence for three dimensional reconstruction, ${ }^{2,3}$ even for clusters containing up to 70 atoms

\footnotetext{
a) Also at: Department of Chemistry, Northwestern University, Evanston, Illinois 60208
}

( $\sim 10 \AA$ diameter). Since small particles of silicon of $20-30$ $\AA$ diameter have been observed to have crystalline bulklike lattices, ${ }^{14,15}$ obtaining information about the structures of small clusters and the transition to bulklike crystalline or amorphous structure would be extremely important in the study of basic material properties and growth processes.

The paucity of experimental structural information is largely due to the difficulty of preparing monodispersed samples of sufficient quantity and density, and to the difficulty of applying traditional spectroscopy techniques to gas phase clusters. The application of mass spectrometry to molecular and ion beam studies of metal and semiconductor clusters has made it possible to experimentally determine size-dependent behavior on an atom-by-atom scale. In addition, a great deal of information about very small clusters, mostly metal dimers and trimers, has been obtained by matrix isolation experiments. ${ }^{16}$ And many properties of small particles have been elucidated by studies on substrate supported particles, albeit with wide size distributions. ${ }^{17,18}$

Combining mass spectrometry with cluster deposition is technically very difficult, but makes new experiments on size-selected clusters possible. The first experiments of this kind were done by Wöste and co-workers, who studied the 


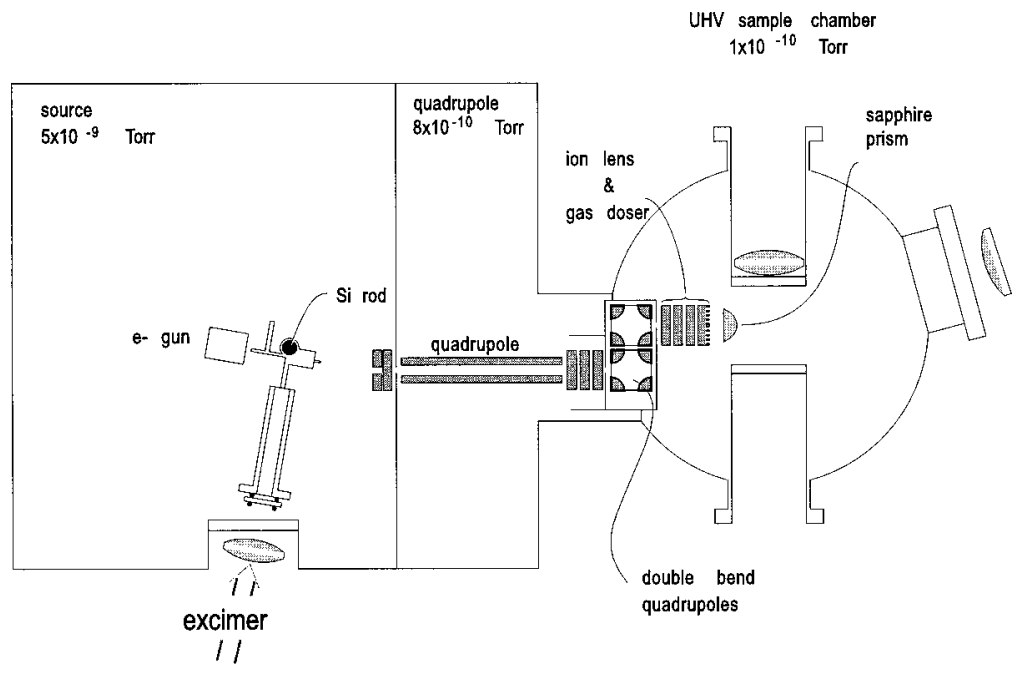

FIG. 1. Schematic diagram of silicon cluster deposition apparatus.

photographic properties of small $\mathrm{Ag}_{n}$ clusters. ${ }^{19}$ Subsequently, DiCenzo, Berry and Hartford recorded photoemission spectra for size-selected gold clusters deposited on amorphous carbon. ${ }^{20}$ Further photoemission studies on sizeselected clusters were performed by Eberhardt et al. ${ }^{21} \mathrm{Har}-$ bich, Lindsay and co-workers have done a number of studies on size-selected, matrix-isolated clusters, ${ }^{22,23}$ while Jarrold and co-workers have done several experiments on sizeselected and deposited silicon clusters. ${ }^{24-26}$ In a recent paper, Moskovits and co-workers identified the structure of $\mathrm{Ag}_{5}$ deposited in an argon matrix using Raman spectroscopy. ${ }^{27}$

The relatively low flux of cluster ions in these experiments results in small quantities of deposited material within a reasonable amount of time. In the experiments reported here, a typical deposition rate for $\mathrm{Si}_{6}^{+}$was $1.5 \AA$ per hour. We have used surface plasmon-polariton (SPP) enhanced Raman spectroscopy to study the clusters, ${ }^{28,29}$ a technique which is capable of detecting molecular species at monolayer surface coverages. Here we report on Raman spectroscopy studies of several small cluster sizes: $\mathrm{Si}_{4}, \mathrm{Si}_{6}$ and $\mathrm{Si}_{7}$. As described in an initial communication, ${ }^{30}$ the sharp, wellresolved vibrational spectra were found to be in good agreement with predicted Raman frequencies and intensities from $a b$ initio structure calculations, making it possible to assign the structures of these small clusters.

In addition to the properties of the isolated clusters, we describe some preliminary observations of interactions between silicon clusters. Since most conceivable technological applications of clusters would involve clusters deposited on a support or assembled in some way to form new materials, cluster-substrate and cluster-cluster interactions are important areas of study. Cluster deposition or aggregation has been used to achieve different growth kinetics and hence different material properties. ${ }^{31,32}$ Using Raman spectroscopy, we have examined the aggregation properties of small silicon clusters under two sets of conditions: warming the matrix to allow the clusters to diffuse in the matrix, and sublimating the matrix completely to "soft land" the clusters onto a substrate. We find that small silicon clusters aggregate under these very gentle conditions, even the relatively stable, highly symmetric ones. The resulting Raman spectra of the aggregates are independent of initial cluster size and for both methods of preparation bear some similarities to those of amorphous silicon.

\section{EXPERIMENT}

\section{A. Production and size-selection of silicon cluster ions}

Figure 1 shows a diagram of the experimental apparatus for depositing size-selected clusters. The apparatus is essentially the same as that used for previous studies of sizeselected deposited clusters ${ }^{25,26}$ with modifications to do the in-situ SPP enhanced Raman measurements and increase the stability of the ion beam. A pulsed laser-ablation source produced the silicon cluster ions (see Fig. 2). A Lambda Physik excimer laser (EMG103MSC) operating at $308 \mathrm{~nm}$ (xenon chloride) between 100 and $200 \mathrm{~Hz}$ ablates silicon from a rotating and translating polycrystalline target rod $(99.999 \%$ purity) into a continuous flow ( $\left.\sim 25 \mathrm{~cm}^{3} / \mathrm{s}\right)$ of He buffer gas $(99.9999 \%)$ at $13-20$ Torr. The production of positive cluster ions was enhanced by preionizing the He buffer gas with a $1 \mathrm{keV}$ electron beam (emission current $5 \mathrm{~mA}$ ) fired into the He gas through a pinhole upstream of the silicon target rod. The injected electrons are also crucial for effective ion transport because the electrons neutralize space-charge effects and reduce the expansion of the ion beam. After a mild ex-

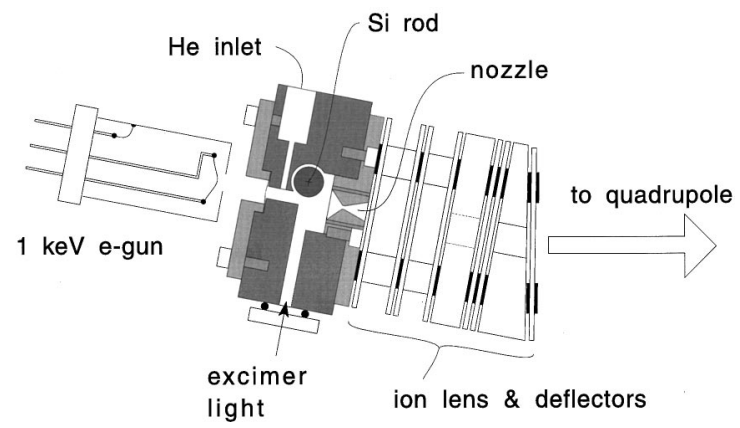

FIG. 2. Laser vaporization source used to produce the silicon clusters. An electron gun at $1 \mathrm{keV}$ produces a $\mathrm{He}$ plasma and the clusters are ionized by charge exchange. See text for details. 


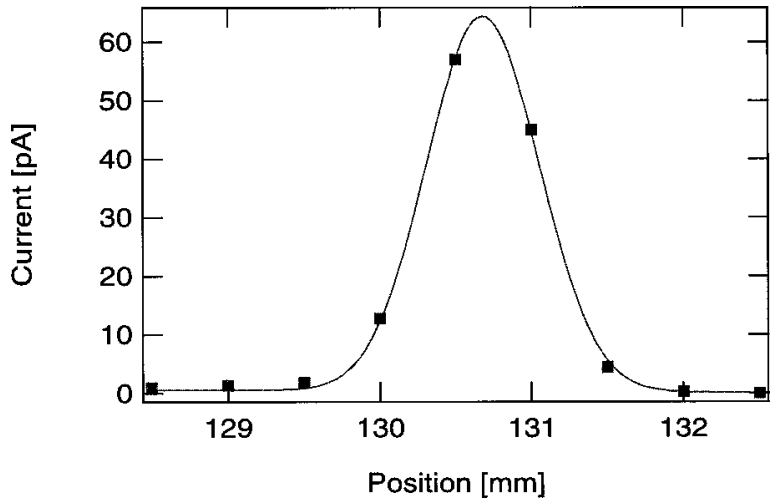

FIG. 3. Current profile measured on a $0.5 \mathrm{~mm}$ wire moved perpendicularly to the beam. The continuous line is a fit to the data taking into account the thickness of the wire and assuming a Gaussian beam profile. This yields a diameter for the cluster beam of $0.85 \mathrm{~mm}$ full width at half maximum (FWHM).

pansion into a low-vacuum chamber (base pressure 5 $\times 10^{-9}$ Torr, operating pressure $6 \times 10^{-4}$ Torr), the cluster ions are focused into a low energy ion beam, pass into a differentially pumped chamber and are injected into a quadrupole mass spectrometer in a medium vacuum environment (base pressure $8 \times 10^{-10}$ Torr, operating pressure 6 $\times 10^{-7}$ Torr). The cluster ions are mass filtered, focused through an aperture, and then undergo a double right-angle bend into the ultrahigh vacuum (UHV) deposition chamber which is cryo- and turbo-pumped to a base pressure of 7 $\times 10^{-11}$ Torr (operating pressure $5 \times 10^{-8}$ Torr of He). The double right-angle bend deflects the clusters out of the path of any residual effusive flow from the low and medium vacuum chambers that might contaminate the sample during deposition.

Immediately before the deposition substrate, the cluster ions are slowed to their final deposition energy while being focused with a three element zoom lens. Typically, $27 \mathrm{eV}$ was used as the final deposition energy, with some depositions done at $63 \mathrm{eV}$. Typical cluster ion currents measured at the substrate holder ranged from 120 up to $800 \mathrm{pA}$ for $\mathrm{Si}_{6}^{+}$in a spot varying between 0.8 to $3.5 \mathrm{~mm}$ in diameter. Deposition times of 1-3 hours yielded the equivalent of $0.5-1$ monolayers of clusters distributed in the matrix. In early experiments, Rutherford backscattering (RBS) was used to calibrate cluster deposition rates and measure the spot size. In later experiments, the spot could be directly observed by imaging the shift in the surface plasmon polariton resonance angle as well as measuring the current profile directly, as shown in Fig. 3.

\section{B. Deposition and matrix isolation}

The matrix gas to be co-condensed with the clusters was directed through an element which served as both the final ion optic and a gas diffuser. The substrate was a hemicylindrical sapphire prism, cooled by a continuous flow liquid $\mathrm{He}$ cryostat to between 8 and $15 \mathrm{~K}$. The prism was positioned 5 $\mathrm{mm}$ in front of the final ion optic/gas diffuser as a compromise between maintaining a small cluster beam spot size and ensuring a uniform thickness of the matrix. First, a buffer layer of $50 \AA$ was deposited to ensure that no cluster came into contact with the silver film, then the cluster-containing matrix was built to a thickness of typically 500-1500 $\AA$. After the clusters were deposited, a layer of pure matrix material was deposited to form a "cap" several microns thick, providing the necessary optical conditions for the plasmon polariton, and isolating the clusters from subsequent contamination. Optical interferometry was used to calibrate the deposition rate of the matrix, while the position of the SPP dip was used to calibrate its index of refraction.

The cluster concentration in the matrix was controlled by monitoring the cluster current and regulating the deposition rate to produce the desired concentration. The cluster ions were neutralized in the matrix by electrons generated from a shielded hot filament $(+4 \mathrm{eV}$ to ground, 3, $\mu \mathrm{A}$ emission current). Solid $\mathrm{N}_{2}$ was used as the matrix material for most of the studies. Surface chemistry and gas phase cluster reactivity experiments have shown that $\mathrm{N}_{2}$ is unreactive with bulk silicon surfaces and silicon clusters at low temperature. ${ }^{33}$ Matrices of $\mathrm{Kr}$ with $1 \% \mathrm{~N}_{2}$, or Ar with $1 \%$ $\mathrm{SF}_{6}$, were used for some of the experiments. Spectra recorded in the different matrices were not significantly different.

\section{SPP enhanced Raman spectroscopy of matrix- isolated silicon clusters}

SPP enhanced Raman spectroscopy ${ }^{19,20,34}$ was used to characterize the vibrational properties of the clusters. The sample geometry and Raman optics configuration are shown in Fig. 4. The excitation laser was an argon ion laser operating at between 514.5 and $457.94 \mathrm{~nm} \mathrm{(10-200} \mathrm{mW,}$ $p$-polarized) with spatial and plasma line filters. The beam was focused by a cylindrical lens to $100 \mu \mathrm{m} \times 2 \mathrm{~mm}$. This leads to $\sim 10^{11}$ clusters in the laser spot. A re-entrant quartz viewport provided access for the $F 2$ collection optics at $\sim 55 \mathrm{deg}$ from normal on the Ag side of the prism. The scattered light collimated by the collection lens was focused by an $f$-matching lens into a triple grating spectrometer (SPEX Triplemate) with an $1800 \mathrm{gr} / \mathrm{mm}$ grating final stage, providing approximately $1 \mathrm{~cm}^{-1} /$ pixel dispersion onto a backthinned, $\mathrm{LN}_{2}$ cooled, Tektronix $512 \times 400$ charge coupled device (CCD) detector (Princeton Instruments). The resolution of the spectrometer was approximately $3 \mathrm{~cm}^{-1}$. Exposure times were typically $1200 \mathrm{sec}$, with multiple spectra averaged together. A $3 \times 3$ median filter was used to remove cosmic rays from unbinned images. The images were then summed along the nondispersive CCD columns to obtain a 2-D spectrum of intensity versus Raman energy loss. Coarse alignment of the substrate and optics was done with two intersecting laser beams through the chamber. The Raman signal from the $\mathrm{N}_{2}$ matrix or $\mathrm{N}_{2}$ or $\mathrm{SF}_{6}$ molecular species, seeded in the $\mathrm{Kr}$ or Ar matrices, respectively, was used to optimize the alignment and as an in situ calibration of the Raman cross sections of the clusters. The unenhanced Raman lines from the $\mathrm{Al}_{2} \mathrm{O}_{3}$ substrate were attenuated by the Ag film (by $\sim 1 / 200$ ), but were still detected and used to calibrate the vibrational energy scale. ${ }^{35}$

In our experiments, the surface-plasmon-polariton (SPP) was excited using the attenuated total reflection (ATR), or 

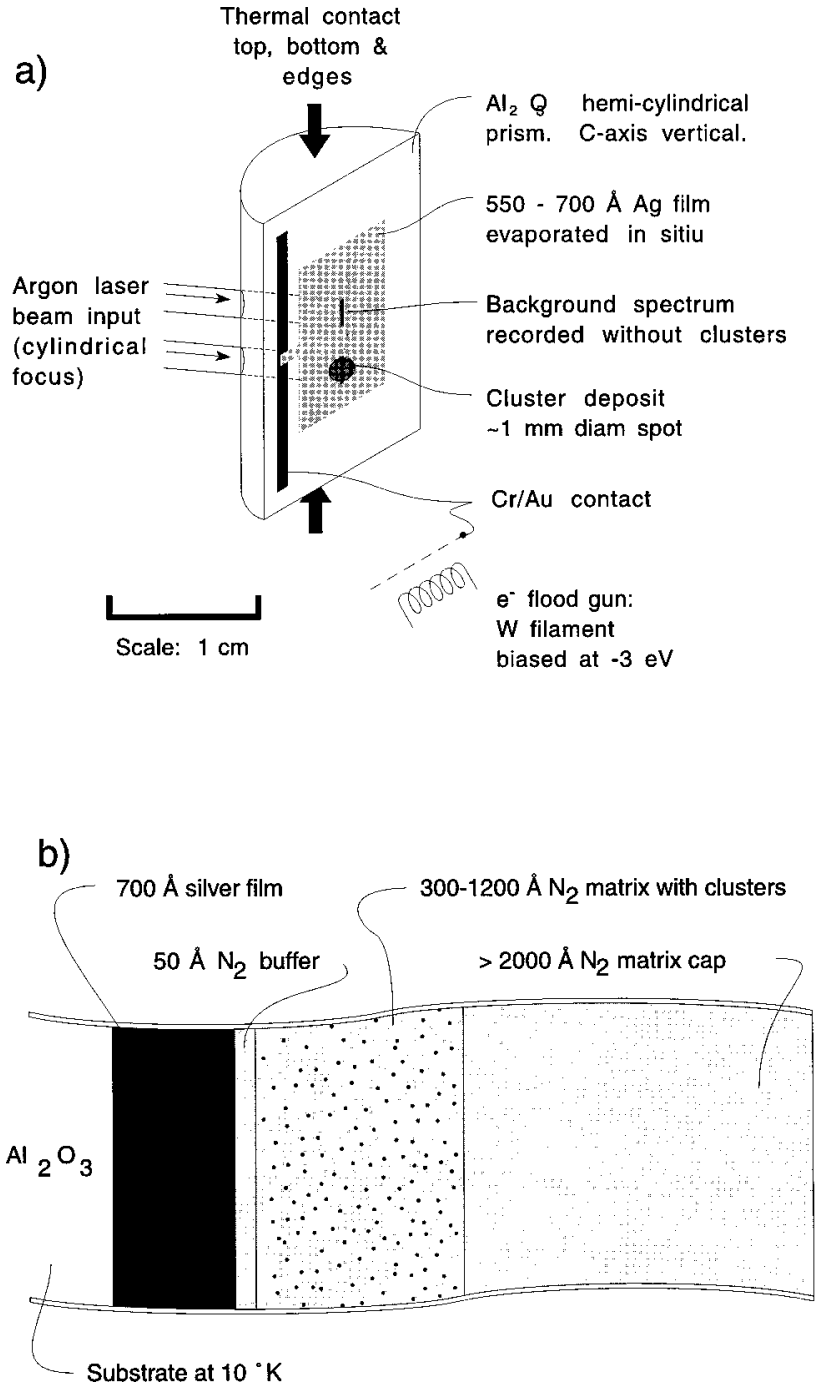

FIG. 4. Schematic of substrate configuration, showing prism, Ag, cluster/ matrix and capping layers, and incident and collected light angles.

Kretschmann configuration. In this arrangement, SPP modes of a metal film deposited on a dielectric prism are excited by adjusting the incident angle of the laser beam. This matches the laser wave vector to that of the nonradiative branch of the dispersion curve of the surface-plasmon-polariton at the metal/prism interface. Approximately $550 \AA$ of $\mathrm{Ag}$ was deposited in-situ on the sapphire prism, with the clustercontaining matrix deposited on the Ag film. (Later this was increased to $700-750 \AA$ in order to further attenuate the sapphire substrate Raman lines.) The lower refractive index of the matrix relative to the prism forms an asymmetric slab configuration that causes the nonradiative SPP field to decay exponentially from the $\mathrm{Ag} /$ matrix interface. The resonant nature of the SPP excitation causes the field intensity at the $\mathrm{Ag} /$ matrix interface to be a factor of $\sim 50-100$ times the incident laser intensity. This enhancement and exponential localization of the incident optical field to a volume (by definition) comparable to the sample size makes possible the large and selective enhancement of the Raman signal of our samples.

The enhanced field strength decreases exponentially with distance from the $\mathrm{Ag} /$ matrix interface with a calculated 1/e distance of $\sim 700 \AA$. The calculated enhancement was found to depend critically on the thicknesses and optical constants of the matrix and Ag film. We found that the details of preparing the Ag film produced observable differences in the resonance condition and enhancement. The largest SPP enhancement and narrowest dip (see below) were found for $\mathrm{Ag}$ films deposited at $200 \mathrm{~K}$ and annealed for several hours at room temperature. ${ }^{38}$

Experimentally, we can determine when the conditions for SPP excitation are met by observing the attenuated total reflection of the laser beam from the Ag film, a small temperature rise of the substrate, and a large increase in the Raman signal of the matrix materials. Figure 5(a) is an image of the laser light reflected from the Ag film when the $\mathrm{N}_{2}$ matrix on the film contained $0.8 \% \mathrm{Si}_{4}$. For this image, the incident laser beam was expanded to a diameter of $3 \mathrm{~cm}$ so that the entire length of the sapphire prism could be illuminated at once. The vertical axis of the image is the spatial position along the vertical axis of the sapphire prism. The horizontal axis of the image is the angle at which the laser light was reflected from the $\mathrm{Ag}$ film. The dark line in the image is the angle at which the plasmon polariton is resonantly excited in the $\mathrm{Ag}$ film. Over most of the prism length, the resonant condition is at a constant angle $(49.5 \mathrm{deg})$. In the region where the clusters were deposited in the matrix, however, there is a large shift in the resonance angle, indicating a change in the dielectric constant of the matrix, resulting in a visible dip in Fig. 5. Figure 5(b) shows line sections taken from the image which show the angle, width and depth of the resonance condition both on and off the spot containing the clusters. The line sections show the broadening and reduction in peak intensity of the resonance produced by the absorption from the Si clusters. Most importantly, the resonance condition results in an increase in the observed Raman intensities. Figure 5(c) shows the $2328 \mathrm{~cm}^{-1} \mathrm{~N}_{2}$ matrix ${ }^{36}$ Raman signal versus incidence angle of the laser beam for a pure matrix of $\mathrm{N}_{2}$ and a matrix with $0.2 \%$ concentration by volume $\mathrm{Si}_{4}$. The solid line through the experimental points is from calculations of the integrated field intensity using the Fresnel equations for a multilayer structure with complex dielectric constants. The optical constants of Johnson and Christy were used for Ag. ${ }^{37}$ The calculated angle of $51 \mathrm{deg}$ and field enhancement of $\sim 90$ for the pure $\mathrm{N}_{2}$ matrix matches the experimental results using an index of refraction of 1.34 for the $\mathrm{N}_{2}$. Similarly, a complex index of $1.2+i 0.003$ for the cluster-containing matrix was found to match the experimentally determined enhancement for this set of deposition conditions. From the cluster concentration and effective medium theory, we can, in principle, extract a cross section for the Raman process for the clusters. In practice, however, it turns out that we would have to take into account many parameters in this estimation, such as the spatial distribution of the clusters, the spatial variation in the intensity of the laser spot, and the shift in the resonance with the concentration of clusters.

Since the clusters were deposited in a small spot $\sim 1-3 \mathrm{~mm}$ in diameter, it was possible to take a background spectrum a few $\mathrm{mm}$ away under virtually identical conditions. This background can then be subtracted from the spec- 
a)

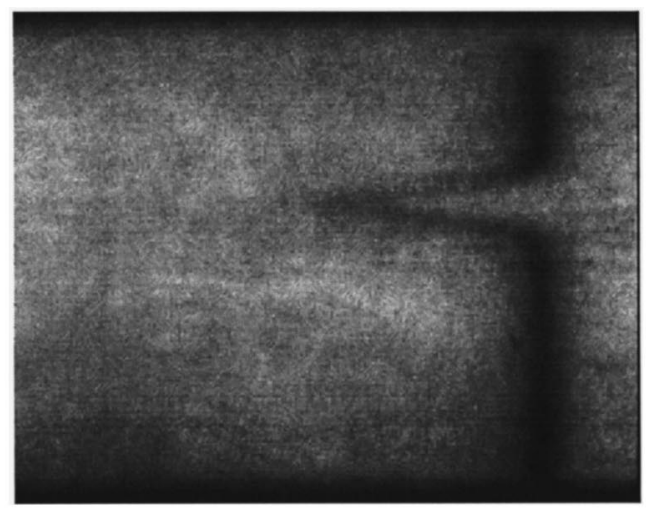

b)

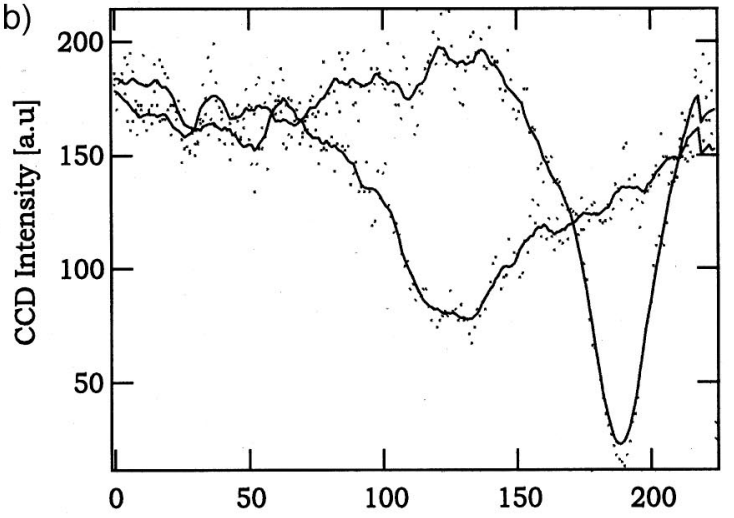

Pixel

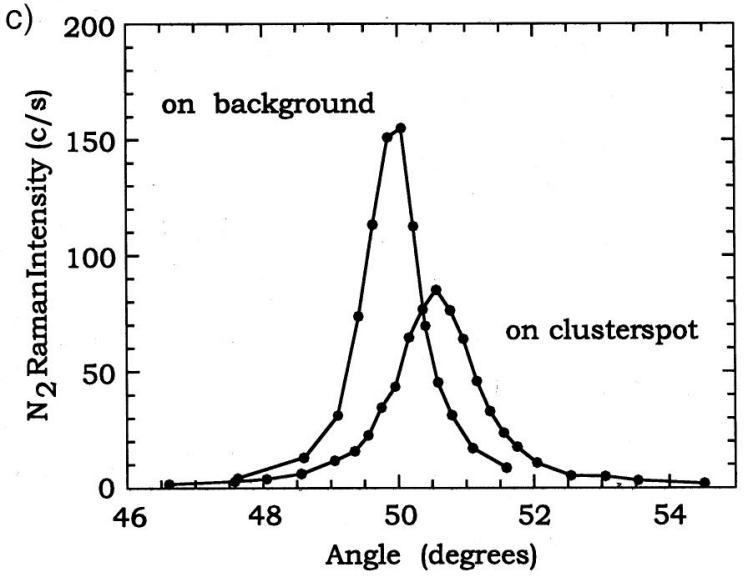

FIG. 5. SPP enhancement: (a) Laser light reflected from the silver film. At the SPP resonance angle the light is not reflected and a dark line appears. The observed dip is the signature of the presence of clusters in the matrix, which slightly shifts the SPP resonance angle. (b) Line section taken from the image above. (c) $\mathrm{N}_{2}$ signal versus angle for both neat matrix and matrix with clusters. The solid lines through the data points were calculated with different matrix indices of refraction, including a small imaginary part for cluster containing matrix.

trum taken at the cluster position. In addition to the Raman lines from the $\mathrm{Al}_{2} \mathrm{O}_{3}$ substrate, the background consisted of broadband surface plasmon-polariton emission, stray light, fluorescence and possibly Raman scattering from contaminants on the Ag film or in the matrix. The latter were typically derived from hydrocarbons (as identified by their characteristic Raman bands) and were minimized by baking the vacuum system and depositing a fresh $\mathrm{Ag}$ film prior to each cluster deposition run. In later experiments, the hydrocarbon contamination and the accompanying Raman and luminescence background were virtually eliminated by a thorough cleaning of the chambers, the cryotrapping of the diffusion pumps on the low and medium vacuum chambers, and the installation of a gate valve which allowed the low vacuum chamber to be isolated from the other chambers except during cluster deposition.

\section{RESULTS AND DISCUSSION}

\section{A. Raman spectra of small clusters}

Figures 6(a)-6(c) show SPP enhanced Raman spectra from 180 to $560 \mathrm{~cm}^{-1}$ for $\mathrm{Si}_{4}, \mathrm{Si}_{6}$ and $\mathrm{Si}_{7}$, respectively. The predicted geometries, frequencies and Raman intensities from $a b$ initio calculations are shown in the figure for comparison. For each cluster size, the experimental spectrum shown is an average of several exposures and cluster depositions. Typical conditions for the spectra shown were $\sim 0.1 \%$ cluster concentration by volume and an $\mathrm{N}_{2}$ Raman intensity of 200-300 cps; aggregation begins to occur for concentrations higher than $0.1 \%$. The higher Raman intensities measured for $\mathrm{Si}_{4}$, compared to $\mathrm{Si}_{6}$ and $\mathrm{Si}_{7}$, may suggest an additional enhancement arising from electronic resonance effects. Numerous attempts to measure the Raman signature of $\mathrm{Si}_{5}$ and $\mathrm{Si}_{10}$ were unsuccessful, presumably because of low Raman cross sections and a limited signal-to-noise ratio. A background spectrum and slowly varying component have been subtracted to increase the visibility of the sharp cluster lines. No lines attributed to the clusters were observed at higher frequencies and the experimental conditions did not permit measurements at significantly lower frequencies.

For $\mathrm{Si}_{4}, \mathrm{Si}_{6}$ and $\mathrm{Si}_{7}$ the observed lines are all less than $600-650 \mathrm{~cm}^{-1}$. The Raman spectra of bulk crystalline silicon consist of a single line around $520 \mathrm{~cm}^{-1}$, while the spectrum for amorphous silicon is broad and reflects the density of states. ${ }^{39}$ The multiple, sharp lines observed for the small clusters are indicative of their molecular nature and demonstrate that these clusters cannot be understood in terms of bulk silicon properties.

\section{B. Effect on Raman intensities from proximity to metal surface}

The intensities of the scattered Raman signal are significantly affected by the clusters' proximity to the metal film. These "surface selection rules" can result in large modedependent differences in the observed intensities compared to the standard 90 deg scattering geometry. The intensity versus angle of a radiating dipole close to the surface of a good conductor can be qualitatively understood in terms of image dipoles [Fig. 7(a)]. For dipoles oriented parallel to the surface of the metal, the radiation from the image is out of phase with that of the real dipole, resulting in little or no observed radiation. Alternately, for a dipole oriented perpendicular to the Ag surface, the fields from the real and image dipoles add constructively, although the radiation pattern of the dipoles results in a strong angular dependence of the observed radiation.

For the case of a molecule directly on a metal/vacuum interface, intensities and angular dependencies have been ex- 

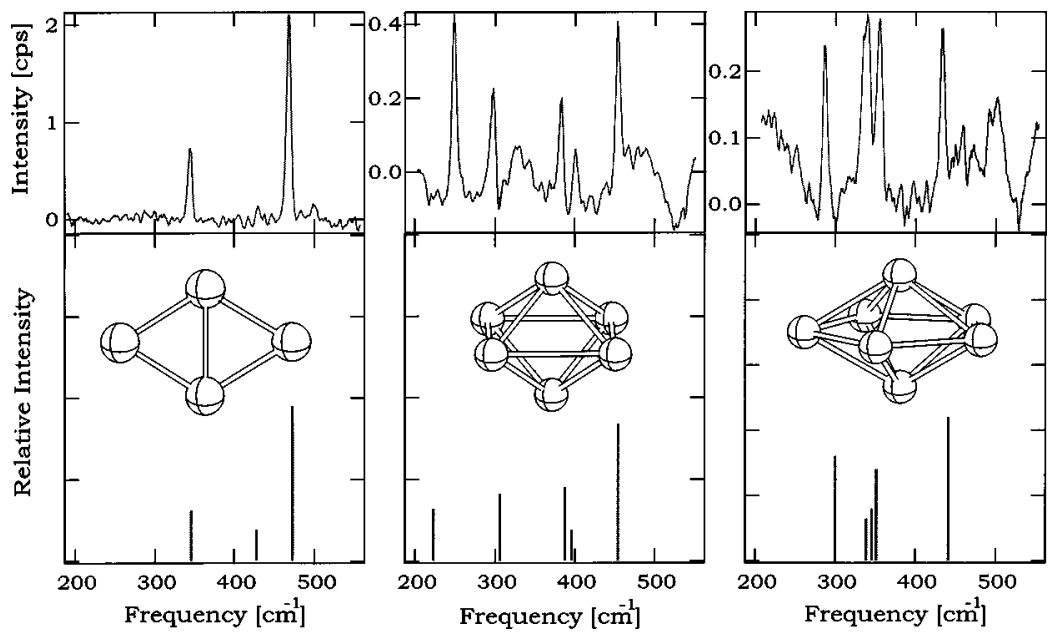

FIG. 6. Measured spectra of $\mathrm{Si}_{4}, \mathrm{Si}_{6}$, and $\mathrm{Si}_{7}$, predicted structures, and predicted spectra. plicitly calculated by several researchers, including Greenler and Slager, ${ }^{40}$ Moskovits ${ }^{41}$ and Campion. ${ }^{42}$ With some modifications, we can use these methods to compare Raman intensities for a standard $90 \mathrm{deg}$ scattering geometry to the case of clusters distributed in a matrix at a finite distance from an Ag film. The geometry for calculating the radiated intensity from a dipole $P$, in a matrix of index $n_{1}$, a distance $z$ above an Ag surface, is shown schematically in Fig. 7(b). The calculation relies on finding the combined response of the wave directly radiated from the dipole and the wave reflected from the metal surface. The dipole radiation pattern and the polarization and angle-dependent phase shift of the reflected wave give rise to variations in intensity for different angles of reflection and dipole orientations. For dipoles radiating visible wavelength light above an Ag surface, the greatest intensity

a)

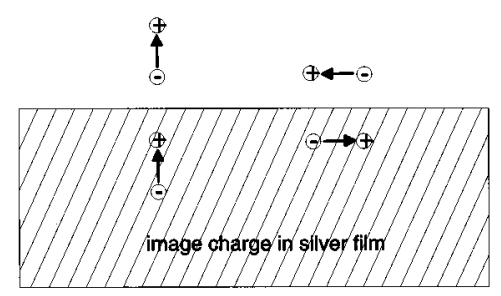

b)

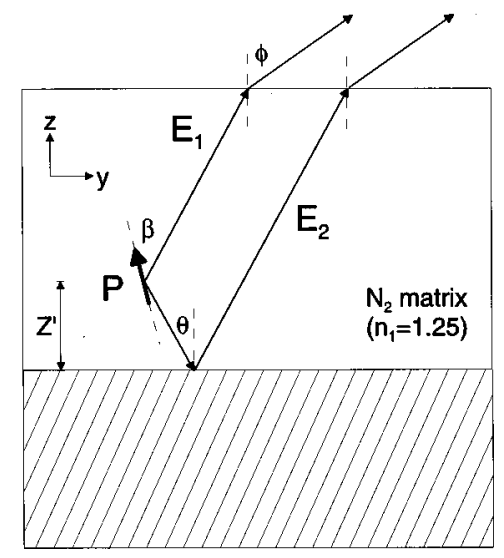

FIG. 7. Surface selection rules: (a) Dipoles above a metal surface. (b) Geometry for calculating the radiated intensity from a dipole near a metal surface (see text). comes from $z$ oriented dipoles, with the maximum integrated intensity coming from around $60 \mathrm{deg}$ from normal. ${ }^{41-43}$

Since these effects have been worked out in detail by others, we limit our discussion to the consequences of the clusters being distributed in a matrix rather than located exactly at a metal/vacuum interface. This results in an additional phase shift for the reflected wave because of the longer path,

$$
\Delta s=\frac{z(1+\cos 2 \theta)}{\cos \theta} .
$$

Since the SPP field falls off exponentially over a distance which is less than a wavelength, the radiated intensity will be dominated by those dipoles which are closest to the Ag surface. Compared to the case of the molecule directly on the metal/vacuum interface, this additional phase shift has the effect of slightly increasing the relative intensity of the dipoles which are surface-parallel while slightly decreasing the intensity of those that are surface-normal.

Refraction of the radiated light at the matrix/vacuum interface must also be included in our calculation. Assuming this interface is parallel to the Ag film and the matrix layer is of sufficiently high quality that there is negligible scattering, the external angle $\phi$ at which the radiated light is observed is related to the internal angle $\theta$ by Snell's Law, $\sin \phi$ $=n_{1} \sin \theta$. For a matrix of index $n_{1}=1.25$, the radiated light at an internal angle larger than $\sim 53 \mathrm{deg}$ is totally internally reflected at the matrix/vacuum interface.

With these effects included, we have calculated the relative intensities versus external angle for $x, y$ and $z$ oriented dipoles distributed in the matrix. The intensities were integrated over the $z$ direction with the contributions for the dipoles within a thickness $d z$ weighted by an exponential factor reflecting the decay of the SPP excitation field with distance away from the Ag/matrix interface. Since the SPP electric field is oriented in the $z$ direction, the Raman intensities are related to the $\alpha_{x z}, \alpha_{y z}$ and $\alpha_{z z}$ components of the polarizability tensor. Averaging over all orientations of the clusters yields the intensities in terms of the invariants of the polarizability tensor, $\bar{\alpha}$ and $\gamma^{2}$, the isotropic and anisotropic parts respectively. ${ }^{43,44}$ Integrating over the range of angles 
TABLE I. Vibrational parameters for silicon clusters.

\begin{tabular}{ccccccc}
\hline \hline Cluster & $\begin{array}{c}\text { Experimental } \\
\text { frequency } \\
\mathrm{cm}^{-1}\end{array}$ & $\begin{array}{c}\text { Calculated }^{\mathrm{a}} \\
\text { frequency } \\
\mathrm{cm}^{-1}\end{array}$ & $\begin{array}{c}\text { Absolute } \\
\text { deviation } \\
\mathrm{cm}^{-1}\end{array}$ & $\begin{array}{c}\text { Symmetry } \\
\text { assignment }\end{array}$ & $\begin{array}{c}\text { Calculated }^{\mathrm{b}} \\
\text { intensity } \\
\left(90^{\circ}\right)\end{array}$ & $\begin{array}{c}\text { Corrected }^{\mathrm{c}} \\
\text { intensity }^{\circ}\end{array}$ \\
\hline $\mathrm{Si}_{4}$ & 345 & 346 & 1 & $a_{g}$ & 1.0 & 3.1 \\
& 428 & & $b_{3 g}$ & 0.6 & 1.9 \\
$\mathrm{Si}_{6}$ & 470 & 473 & 3 & $a_{g}$ & 7.0 & 9.5 \\
& 252 & 223 & 29 & $b_{2 g}$ & 1.0 & 3.2 \\
& 300 & 306 & 6 & $a_{1 g}$ & 1.7 & 4.1 \\
& 386 & 388 & 2 & $b_{1 g}$ & 1.4 & 4.5 \\
& 404 & 396 & 8 & $e_{g}$ & 0.6 & 1.9 \\
$\mathrm{Si}_{7}$ & 458 & 454 & 4 & $a_{1 g}$ & 8.2 & 8.4 \\
& 289 & 300 & 11 & $e_{2}^{\prime}$ & 1.0 & 3.2 \\
& 340 & 339 & 1 & $e_{2}^{\prime}$ & 0.4 & 1.3 \\
& 340 & 346 & 6 & $e_{1}^{\prime \prime}$ & 0.5 & 1.6 \\
& 358 & 352 & 6 & $a_{1}^{\prime}$ & 1.4 & 2.8 \\
& 435 & 441 & 6 & $a_{1}^{\prime}$ & 4.3 & 4.4 \\
\hline \hline
\end{tabular}

${ }^{\mathrm{a}} \mathrm{QCISD} / 6-31 \mathrm{G}^{*}$ frequencies for $\mathrm{Si}_{4}$ and $\mathrm{Si}_{6}$, and MP2/6-31G* frequencies for $\mathrm{Si}_{7}$, all scaled down uniformly by $5 \%$. See text.

${ }^{\mathrm{b}}$ Relative intensities at the $\mathrm{HF} /(7 s, 6 p, 2 d)$ level of theory (see text) for a 90 deg scattering geometry assuming no electronic resonance effects.

${ }^{c}$ The calculated intensities corrected for the experimental geometry effects (see text).

subtended by the light collection lens, and using appropriate values of the dielectric constants for the experimental conditions $^{45}$ of the spectra shown in Fig. 6, the calculated intensity is

$$
I \sim \bar{\alpha}^{2}+0.5 \gamma^{2}
$$

Therefore, the intensity observed for each Raman active vibrational mode may be enhanced or suppressed relative to the standard 90 deg scattering geometry where $I \sim \bar{\alpha}^{2}$ $+(7 / 45) \gamma^{2}$.

\section{Theory}

The ground state structures of $\mathrm{Si}_{3}-\mathrm{Si}_{7}$ have been carefully investigated by $a b$ initio quantum chemical techniques as well as by density functional methods. ${ }^{5-7}$ In a previous work, ${ }^{30}$ we reported the vibrational frequencies of $\mathrm{Si}_{4}, \mathrm{Si}_{6}$, and $\mathrm{Si}_{7}$ at the MP2 level (second-order Møller-Plesset perturbation theory) with the polarized $6-31 \mathrm{G}^{*}$ basis set. ${ }^{46} \mathrm{We}$ have now refined the the structure and vibrational frequencies of $\mathrm{Si}_{4}-\mathrm{Si}_{6}$ with the more accurate QCISD (quadratic configuration interaction $)^{46}$ method using the same $6-31 \mathrm{G}^{*}$ basis set. The Raman-active QCISD/6-31G* frequencies for $\mathrm{Si}_{4}-\mathrm{Si}_{6}$, along with the MP2/6-31G* values for $\mathrm{Si}_{7}$, have been uniformly scaled down by $5 \%$ and listed in Table I. The scaling takes into account the systematic overestimations present in the theoretical calculations due to the neglect of anharmonicity effects. The nature of the vibrations can be seen from the associated normal modes which are shown in Figs. 8-10.

The evaluation of Raman intensities requires the use of much larger basis sets. We have evaluated the Raman intensities using Hartree-Fock calculations with a large contracted basis set containing $7 s, 6 p, 2 d$ functions on each silicon atom (derived from McLean and Chandler's contracted $6 s, 5 p$ basis set by addition of a set of diffuse $s p$ functions and two sets of $d$ functions). ${ }^{47}$ The theoretical intensities which correspond to a $90 \mathrm{deg}$ scattering geometry are then corrected for the experimental geometry effects and the resulting relative intensities are shown along with the experimental spectra in Fig. 6.

The predicted ground state structure of $\mathrm{Si}_{4}$ is a planar rhombus with $D_{2 h}$ symmetry. The perimeter bond length at the QCISD/6-31G* level is $2.31 \AA$, while the distance along the short diagonal is slightly larger $(2.40 \AA)$. The rhombus has three allowed Raman lines at $346\left(a_{g}\right), 428\left(b_{3 g}\right)$ and $473\left(a_{g}\right) \mathrm{cm}^{-1}$. The frequencies and relative intensities of the
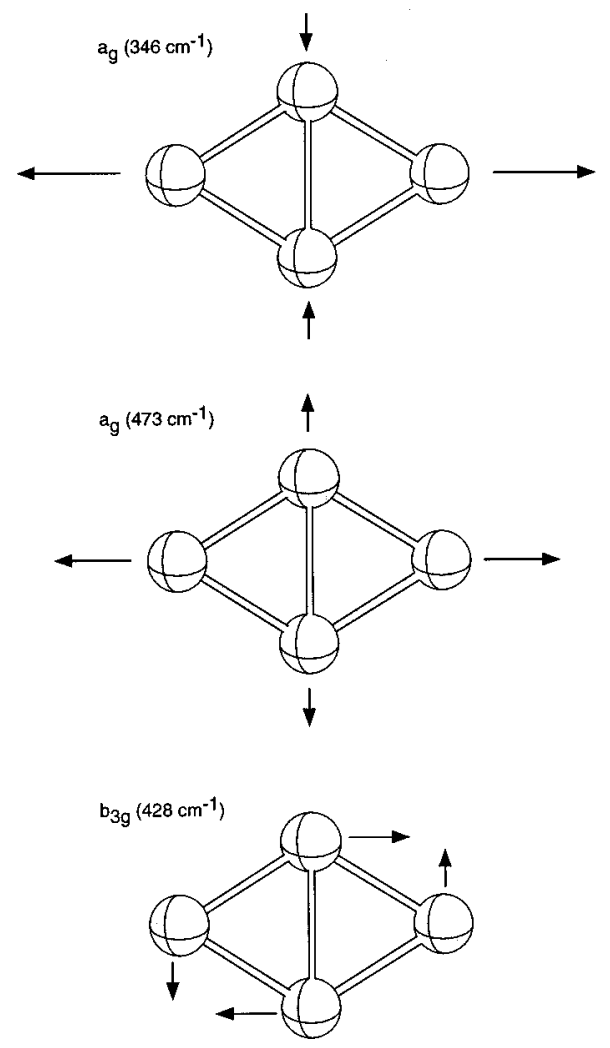

FIG. 8. Normal modes for the three Raman-active vibrations in $\mathrm{Si}_{4}\left(D_{2 h}\right)$. 

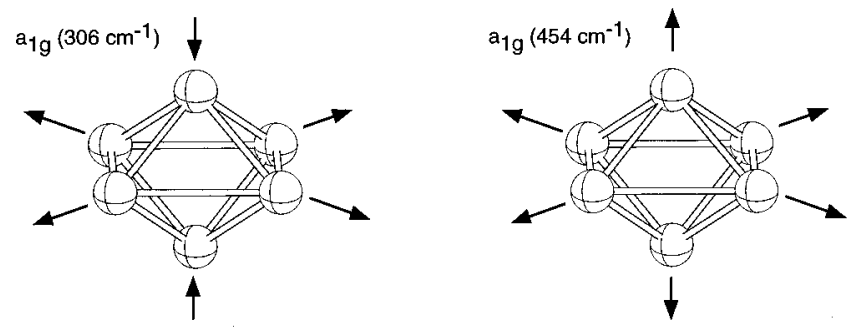

$b_{2 g}\left(223 \mathrm{~cm}^{-1}\right)$

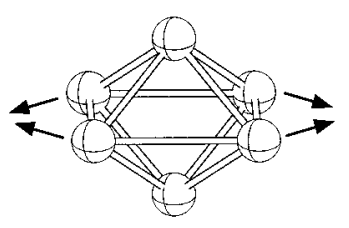

$b_{1 g}\left(388 \mathrm{~cm}^{-1}\right)$

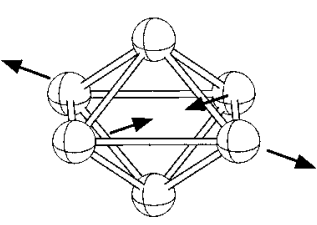

$e_{\mathrm{g}}\left(396 \mathrm{~cm}^{-1}\right)$

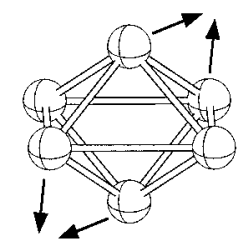

FIG. 9. Normal modes for the five Raman-active vibrations in $\operatorname{Si}_{6}\left(D_{4 h}\right)$.

two $a_{g}$ modes are in essentially perfect agreement with the experimentally observed modes at 345 and $470 \mathrm{~cm}^{-1}$. Inspection of the normal modes (Fig. 8) shows that the higher frequency $a_{g}$ mode $\left(473 \mathrm{~cm}^{-1}\right)$ corresponds to the symmetric breathing vibration for the molecule. This has the highest Raman intensity. The lower frequency $a_{g}$ mode $\left(346 \mathrm{~cm}^{-1}\right)$ corresponds to stretching along one axis and compression along a perpendicular axis, and has a lower Raman intensity. The $b_{3 g}$ vibration predicted at $428 \mathrm{~cm}^{-1}$ has not been observed experimentally, perhaps due to its weak intensity, which makes detection difficult with the existing signal-tonoise. If there is a resonant Raman contribution to the spectrum of $\mathrm{Si}_{4}$, the two $a_{g}$ modes may be enhanced further than the $b_{3 g}$ mode. The $b_{3 g}$ mode has a larger component along the perimeter bonds than the $a_{g}$ modes. The other three vibrational modes of $\mathrm{Si}_{4}$ are not allowed in Raman spectroscopy, though some of them have been seen in matrix infrared spectra. $^{48}$

The ground state structure predicted for $\mathrm{Si}_{6}$ is a distorted octahedron, although the nature of the distortion is difficult to determine due to the shallow nature of the potential energy surface. At the MP2/6-31G* level of theory, which includes electron correlation effects, $\mathrm{Si}_{6}$ has a tetragonal bipyramidal structure with $D_{4 h}$ symmetry. At other levels of theory (such as Hartree-Fock or density functional theory), additional distortions leading to lower symmetries have been found. ${ }^{5-7}$ However, such distortions, which lead to structures such as a bicapped tetrahedron or a capped trigonal bipyramid, involve shallow degrees of freedom and very little energy. We find that the experimental spectra can be conveniently interpreted in terms of the $D_{4 h}$ structure. It can be considered as a strongly compressed octahedron where the two axial atoms are about $2.7 \AA$ apart. Much of the bonding
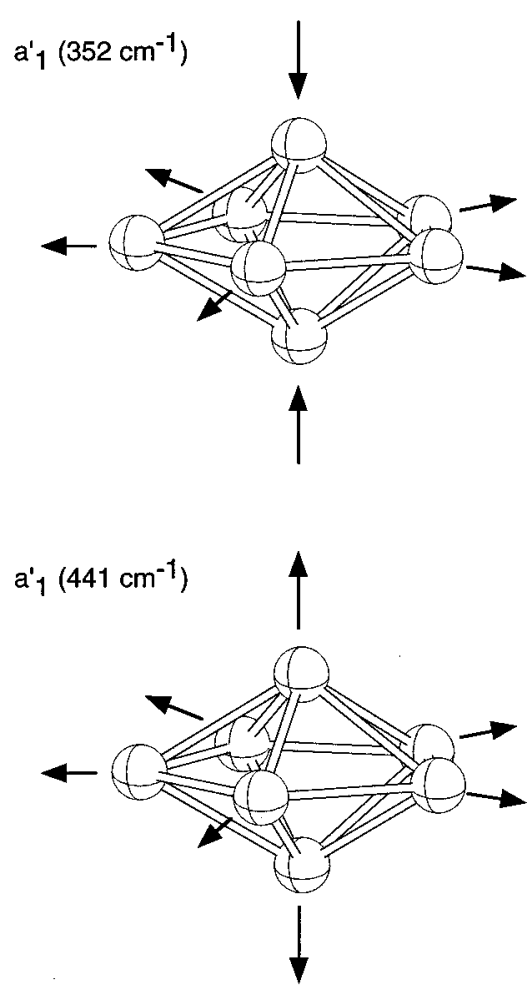

FIG. 10. Normal modes for the two Raman-active $a_{1}{ }^{\prime}$ vibrations in $\mathrm{Si}_{7}$ $\left(D_{5 h}\right)$.

strength in this structure comes from the eight equivalent axial-equatorial bonds $(2.36 \AA)$. The four "bonds"' between the equatorial atoms are about $2.7 \AA$ and do not contribute much to the binding energy. In fact, the distortions involving the weak equatorial bonds are responsible for the shallow energy surface.

At the QCISD/6-31G* level, the $D_{4 h}$ form has five allowed Raman lines at $223\left(b_{2 g}\right), 306\left(a_{1 g}\right), 388\left(b_{1 g}\right), 396$ $\left(e_{g}\right)$, and $454\left(a_{1 g}\right) \mathrm{cm}^{-1}$. With the exception of the $b_{2 g}$ low frequency mode, all other frequencies are within $10 \mathrm{~cm}^{-1}$ of the experimental values. The normal modes corresponding to these frequencies are shown in Fig. 9. As in the case of $\mathrm{Si}_{4}$, there are two $a_{1 \mathrm{~g}}$ modes corresponding to the symmetric breathing vibration $\left(454 \mathrm{~cm}^{-1}\right)$ and the vibration involving elongation and contraction along two perpendicular directions $\left(306 \mathrm{~cm}^{-1}\right)$. Again, the symmetric breathing mode has a higher frequency as well as a higher intensity. The $b_{2 g}$ mode predicted at $223 \mathrm{~cm}^{-1}$ has a low frequency since it involves only the weak equatorial bonds. The degenerate $e_{g}$ mode has a similar normal mode character, though it has a higher frequency $\left(396 \mathrm{~cm}^{-1}\right)$ since it involves the stronger axial-equatorial bonds. It is interesting to note that the $b_{2 g}$ and $e_{g}$ modes result from the splitting of a triply degenerate mode of an octahedron as it distorts to $D_{4 h}$ symmetry. The largest deviation from experiment $\left(29 \mathrm{~cm}^{-1}\right)$ for the $b_{2 g}$ mode may be related to the difficulty in describing the relatively weak equatorial bonds. Finally, the $b_{1 \mathrm{~g}}$ mode at $388 \mathrm{~cm}^{-1}$ is in excellent agreement with the corresponding experimental value $\left(386 \mathrm{~cm}^{-1}\right)$.

$\mathrm{Si}_{7}$ is predicted to have a pentagonal bipyramidal geom- 
etry with $D_{5 h}$ symmetry. As in the case of $\mathrm{Si}_{6}$, it is highly compressed so that the two axial atoms are only $2.51 \AA$ apart (MP2/6-31G*). The equatorial bonds $(2.48 \AA)$ are shorter than the corresponding bonds in $\mathrm{Si}_{6}$. The ten equivalent axial-equatorial bonds $(2.46 \AA)$ are somewhat longer than in $\mathrm{Si}_{6}$. Overall, the $D_{5 h}$ form of $\mathrm{Si}_{7}$ is a compact stable cluster. At the MP2/6-31G* level, it has five allowed Raman lines at $300\left(e_{2}{ }^{\prime}\right), 339\left(e_{2}{ }^{\prime}\right), 346\left(e_{1}{ }^{\prime \prime}\right), 352\left(a_{1}{ }^{\prime}\right)$, and 441 $\left(a_{1}{ }^{\prime}\right) \mathrm{cm}^{-1}$. Figure 10 shows the normal modes of the two $a_{1}{ }^{\prime}$ vibrations. As in the case of $\mathrm{Si}_{4}$ and $\mathrm{Si}_{6}$, the symmetric breathing mode is highest in frequency. However, the two modes in this case appear to be roughly equal in intensity. The normal modes for the other degenerate vibrations of $\mathrm{Si}_{7}$ are more complicated and hence not shown. Experimentally, four lines at $289,340,358$, and $435 \mathrm{~cm}^{-1}$ have been observed, although the width of the $340 \mathrm{~cm}^{-1}$ mode suggests the possibility of two overlapping lines. The agreement between theory and experiment is clearly excellent.

Overall, the agreement between the theoretical frequencies of the calculated ground state structures and the experimental Raman spectra is impressive. Among all the Raman active modes predicted for $\mathrm{Si}_{4}, \mathrm{Si}_{6}$ and $\mathrm{Si}_{7}$, only the relatively weak $b_{3 g}$ mode of $\mathrm{Si}_{4}$ has not been observed. Sharp vibrational lines have been experimentally seen for all other modes with a mean absolute deviation of only $7 \mathrm{~cm}^{-1}$ between theory and experiment. Consideration of other possible structural isomers ${ }^{5-7,30}$ yields qualitative and quantitative results which are completely inconsistent with the experimental spectra. Thus, our results provide convincing evidence for the structural assignments of small silicon clusters as very different from microcrystalline forms.

\section{Fragmentation and/or aggregation of deposited clusters in the matrix}

An important issue in experiments on size-selected clusters is that the deposition process does not result in dissociation of individual clusters and/or aggregation into larger particles. Some of the uncertainties in the deposition process include the redistribution of energy in the deposition and neutralization processes and the exact kinetic energy of the cluster ions prior to deposition due to charging of the matrix. One concern was the possibility that the incident cluster ions would sputter or evaporate matrix material during the deposition or deionization process, since this could dramatically effect the concentration of the clusters in the matrix. However, from optical interference measurements, the thickness of the region of the matrix which contains the clusters is within $1 \%$ of that of the surrounding matrix.

Although many of the details of the deposition process are not completely understood, there is little question that monodisperse cluster samples can be prepared by sizeselected cluster ion deposition. Harbich, Lindsay and coworkers have reported that up to $75 \%$ of $\mathrm{Ag}_{2}^{+}$survive deposition at $30 \mathrm{eV} .{ }^{22}$ As demonstrated in the cluster-surface collision experiments of St. John and Whetten ${ }^{49}$ fragmentation is even less likely for silicon clusters deposited at $25 \mathrm{eV}$, since they have higher dissociation energies and the larger clusters have correspondingly larger heat capacities. ${ }^{3}$ In our
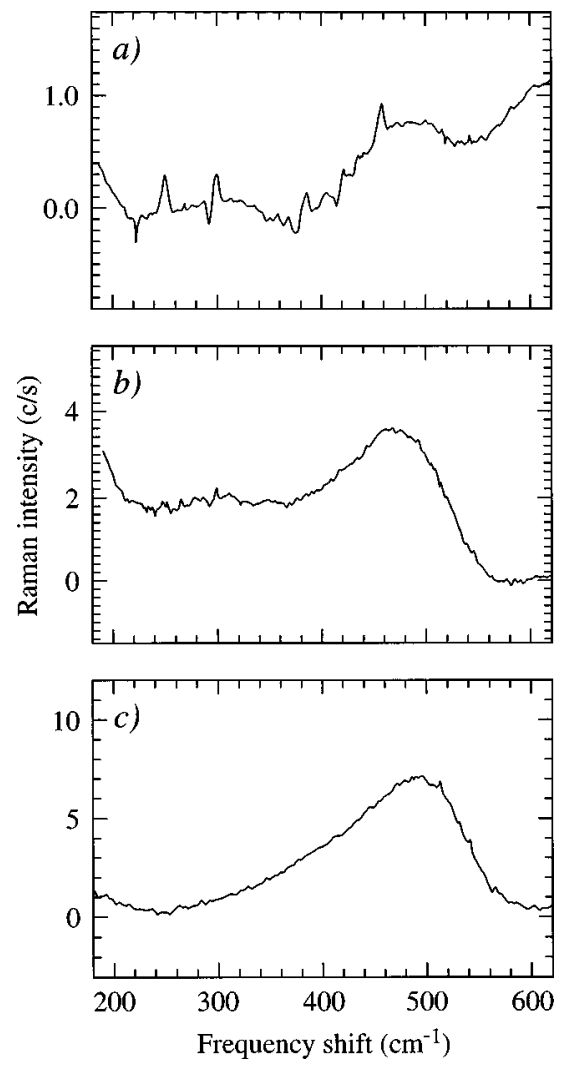

FIG. 11. Cluster aggregation: (a) Raman spectra for $\mathrm{Si}_{6}$ clusters as deposited and showing some aggregation. (b) Spectrum for $\mathrm{Si}_{6}$ matrix aggregated at $T_{\max } \sim 28 \mathrm{~K}$ for several hours. (c) Soft-landed $\mathrm{Si}_{6}$ cluster-deposited film after sublimating the matrix.

experiments, evidence of fragmentation was only observed at the higher deposition energies. For example, $\mathrm{Si}_{10}$ clusters show the Raman signature of $\mathrm{Si}_{4}$ and $\mathrm{Si}_{6}$ clusters at high deposition energies.

\section{E. Matrix aggregation of clusters and cluster- deposited thin films}

While cluster fragmentation can be avoided in the deposition process by using a sufficiently low deposition energy, there is still the possibility of clusters aggregating during the deposition process or once they are in the matrix. We found that it was important to work at low cluster concentrations (around $0.1 \%$ by volume) and low temperatures to avoid coalescence. Aggregation of the clusters was easily identified, because it results in Raman spectra that are independent of the deposited cluster size and very similar to spectra from deposition of much larger cluster "bands," such as $\mathrm{Si}_{25-35}$.

Cluster aggregation was found for relatively high cluster concentrations or if the matrix was annealed for several hours at a temperature just below the point where rapid sublimation occurs. Figure 11 shows several spectra from the same $\mathrm{Si}_{6}$ deposition after raising the temperature, and then recooling to measure the sample under similar conditions. Figure 11(a) shows the as-deposited signal where the prism holder temperature was less than $15 \mathrm{~K}$ for both the deposition and measurements. This deposition was done at relatively high concentration, and as discussed below, the broad peak at $470 \mathrm{~cm}^{-1}$ indicates that some cluster aggregation has 
already occurred. In Fig. 11(b), the spectrum was taken after the temperature was raised to $\sim 28 \mathrm{~K}$ for several hours. ${ }^{50}$ The sharp peaks of the isolated $\mathrm{Si}_{6}$ clusters in Fig. 11(a) are barely visible, replaced with a much broader spectrum due to aggregated clusters. Diffusion of the clusters in the matrix has resulted in the clusters coalescing into larger silicon particles. The Raman cross section for the large silicon particles has increased relative to the isolated clusters because the integrated intensity is much larger. Similar behavior was observed for $\mathrm{Si}_{7}$ : the sharp lines from the isolated clusters evolved into the broad, higher intensity spectrum after warming the matrix to $\sim 29 \mathrm{~K}$ overnight. Some of this increase in intensity could be due to part of the material moving closer to the silver surface.

In the spectra showing coalescence of the small clusters, we were unable to find any evidence of intermediate size aggregates. For instance, when $\mathrm{Si}_{4}$ was deposited at high concentration, only the two sharp lines of the $\mathrm{Si}_{4}$ clusters and the broad spectrum of the aggregate material were observed, with no evidence of $\mathrm{Si}_{8}, \mathrm{Si}_{12}$ or some other relatively small cluster with sharp Raman spectra. Because of its larger cross section compared to the small clusters, we had much greater sensitivity for detection of the aggregated material. The inability to observe the intermediate sizes would suggest that either those clusters have small Raman scattering cross sections, or the aggregation process is unstable, triggering further coalescence once the process has started. For instance, fusion of two $\mathrm{Si}_{6}$ clusters to form $\mathrm{Si}_{12}$ would release roughly $3 \mathrm{eV}$ of energy. ${ }^{3}$ A small fraction of this energy could melt a large volume of matrix, further increasing diffusion and subsequent aggregation of clusters.

By warming the substrate and sublimating the cryogenic matrix, we can "soft land" the clusters on to the Ag surface. ${ }^{31}$ Figure 11(c) shows the Raman spectrum of the matrix-aggregated $\mathrm{Si}_{6}$ clusters deposited as a thin film. This was done by warming the substrate to $60 \mathrm{~K}$ to evaporate the matrix and then redepositing a matrix "cap" to achieve similar enhancement conditions as in Fig. 11(a). Raman spectra from samples prepared in this way are reproducible and independent of the initial cluster size, and the degree of aggregation that has already occurred in the matrix. The Raman intensity has increased further, compared to the isolated small clusters. Part of this enhancement is likely due to the material now being closer to the $\mathrm{Ag} /$ matrix interface and experiencing a larger excitation field strength.

To try to understand the material formed by the aggregation of the clusters, we can compare the spectra to that of bulk amorphous ${ }^{51-53}$ and microcrystalline ${ }^{54}$ silicon and small silicon particles. ${ }^{55}$ Raman spectra of small silicon particles less than $100 \AA$ diameter are similar to those of amorphous silicon, even when the particles are crystalline as determined by transmission electron microscopy (TEM) or x-ray scattering. ${ }^{54,55}$ This has been interpreted in terms of the relaxation of the $k=0$ selection rule due to finite size effects ${ }^{56}$ or to arise from field enhancements of an amorphous surface layer on the microcrystals. ${ }^{57,58}$ Although this means that Raman spectroscopy is not sensitive to the degree of crystalline order of small particles, differences in short-range order of the cluster-assembled materials compared to amorphous sili- con can be identified. Indeed, evidence for thickness dependent Raman spectra of ultrathin films and clusters of Ge has been observed. ${ }^{18}$

The spectra in Figs. 11(b) and 11(c) show some similarities to those of amorphous silicon. The broad peak at $470 \mathrm{~cm}^{-1}$ in the matrix aggregated material and at $490 \mathrm{~cm}^{-1}$ in the cluster-deposited material is similar to the transverseoptic-like (TO) $480 \mathrm{~cm}^{-1}$ peak in amorphous silicon, with the rising edge below $200 \mathrm{~cm}^{-1}$ corresponding to the transverse-acoustic (TA) band. The longitudinal-acoustic (LA) and -optic (LO) region of the spectrum around $300 \mathrm{~cm}^{-1}$ is different for the two cluster-derived materials even though the thickness of the cluster-deposited thin film is only $\sim 1$ cluster diameter. For the matrix-aggregated material, there is more intensity in this region compared to either the cluster-deposited material or bulk amorphous silicon. Given the low temperatures during aggregation and the limited mobility of cluster units compared to individual atoms, it is likely that the material structure is "frozen" into a metastable form.

The matrix aggregated and soft-landed cluster materials appear to have some differences in short-range-order compared to each other and to amorphous silicon. This may be due to finite size effects or the degree of relaxation in the materials. In the work of Lannin and co-workers on Ge clusters and ultrathin films, the TO-like peak shifts and increases in intensity relative to the LA- and LO-like regions as the film thickness increases. ${ }^{18}$ Studies of the width and position of the TO-like peak in amorphous silicon have shown a correspondence with the degree of relaxation of the material. ${ }^{54,59}$ The intensity around $300 \mathrm{~cm}^{-1}$ and the especially broad TO-like peak observed for the matrixaggregated silicon suggest that this material is less relaxed than amorphous silicon prepared by ion implantation or lowtemperature growth. Further characterization of these clusterassembled materials is necessary to elucidate the details of their structures.

\section{CONCLUSIONS}

By depositing size-selected silicon clusters into an inert matrix, we have measured Raman spectra of $\mathrm{Si}_{4}, \mathrm{Si}_{6}$ and $\mathrm{Si}_{7}$, obtaining the first experimental evidence of the detailed structures of silicon clusters containing more than four atoms. We find excellent agreement with predictions from $a b$ initio theory, confirming that these small clusters have compact, close-packed structures which are completely different from bulk microcrystal fragments. Warming the matrix to allow diffusion of the clusters, or complete sublimation of the matrix to "soft land" the clusters, results in irreversible changes in the spectra. Even magic number sizes such as $\mathrm{Si}_{6}$ and $\mathrm{Si}_{7}$ aggregate under these very gentle conditions. The spectra of the resulting cluster-assembled material differ from those of amorphous silicon prepared by conventional methods, but other methods are required to fully characterize the material.

\section{ACKNOWLEDGMENTS}

We thank Edwin Dons for performing the RBS measurements, Eric Bower and Gary Schwartz for assistance with the 
experimental apparatus, David Windt for the program to do multilayer calculations, and Jack Rowe, Lou Brus, Yves Chabal, and Melissa Hines for helpful discussions. We acknowledge the National Science Foundation for partial support of this work. Christian Félix acknowledges the Swiss National Science Foundation for financial support through a Fellowship.

${ }^{1}$ See, for example, M. A. Duncan and D. H. Rouvray, Sci. Am. 261, 110 (1989); Proceedings of the International Symposium on Small Particles and Inorganic Clusters [ Z. Phys. D 26, (1993)]; Proceedings of Faraday Symposium 25 on Large Gas Phase Clusters [ J. Chem. Soc., Faraday Trans. 86, 2343 (1990)].

${ }^{2}$ M. F. Jarrold, Science 252, 1085 (1991), and references therein; J. L. Elkind et al., J. Chem. Phys. 87, 2397 (1987); J. M. Alford et al., Mater. Res. Soc. Symp. Proc. 131, 3 (1989); M. L. Mandich, V. E. Bondybey, and W. D. Reents, J. Chem. Phys. 86, 4245 (1987).

${ }^{3}$ M. F. Jarrold and E. C. Honea, J. Phys. Chem. 95, 9181 (1991).

${ }^{4}$ L. A. Bloomfield, R. R. Freeman, and W. L. Brown, Phys. Rev. Lett. 54, 2246 (1985); W. Begemann, K. H. Miewes-Broer, and H. O. Lutz, ibid. 56, 2248 (1986); M. F. Jarrold and J. E. Bower, J. Phys. Chem. 92, (1988); Q.-L. Zhang, Y. Liu, R. F. Curl, F. K. Tittel, and R. E. Smalley, J. Chem. Phys. 88, 1670 (1988).

${ }^{5}$ K. Raghavachari, Phase Transit. 24-26, 61 (1990); K. Raghavachari and V. Logovinsky, Phys. Rev. Lett. 55, 2853 (1985); K. Raghavachari, J. Chem. Phys. 84, 5672 (1986); K. Raghavachari and C. M. Rohlfing, ibid. 89, 2219 (1988).

${ }^{6}$ P. Ballone, W. Andreoni, R. Car, and M. Parrinello, Phys. Rev. Lett. 60, 271 (1988); D. Tomanek and M. Schluter, Phys. Rev. B 36, 1208 (1987); O. F. Sankey, D. J. Niklewski, D. A. Drabold, and J. D. Dow, ibid. 41, 12750 (1990).

${ }^{7}$ R. Fournier, S. B. Sinnott, and A. E. DePristo, J. Chem. Phys. 97, 4149 (1992).

${ }^{8}$ J. R. Chelikowsky, J. C. Phillips, M. Kamal, and M. Strauss, Phys. Rev. Lett. 62, 292 (1989); ibid. 63, 1649 (1989); J. R. Chelikowsky and J. C. Phillips, Phys. Rev. B 41, 5735 (1990).

${ }^{9}$ For a critical review of bulk empirical potentials applied to silicon clusters, see W. Andreoni and G. Pastore, Phys. Rev. B 41, 10243 (1990), and references therein.

${ }^{10}$ M. F. Jarrold and V. Constant, Phys. Rev. Lett. 67, 2994 (1991).

${ }^{11}$ C. C. Arnold, T. N. Kitsopoulos, and D. M. Neumark, J. Chem. Phys. 99, 766 (1993).

${ }^{12}$ T. N. Kitsopoulos, C. J. Chick, A. Weaver, and D. M. Neumark, J. Chem. Phys. 93, 6108 (1990)

${ }^{13}$ C. C. Arnold and D. M. Neumark, J. Chem. Phys. 99, 3353 (1993).

${ }^{14}$ H. Takagi, H. Ogawa, Y. Yamazaki, A. Ishizaki, and T. Nakagiri, Appl. Phys. Lett. 56, 2379 (1990).

${ }^{15}$ K. A. Littau, P. A. Szajowski, A. J. Mueller, A. R. Kortan, and L. E. Brus, J. Phys. Chem. 97, 1224 (1993)

${ }^{16}$ See, for example, M. Moskovits, in Chemistry and Physics of MatrixIsolated Species, edited by L. Andrews and M. Moskovits (North Holland, Amsterdam, 1989), and references therein.

${ }^{17}$ See, for example, M. G. Mason, Phys. Rev. B 27, 748 (1983); S. B. DiCenzo and G. K. Wertheim, Comments Solid State Phys. 11, 203 (1985); J. Fortner and J. Lannin, Surf. Sci. 254, 251 (1991).

${ }^{18}$ J. Fortner, R. Q. Yu, and J. S. Lannin, J. Vac. Sci. Technol. A 8, 3493 (1990).

${ }^{19}$ P. Fayet, F. Granzer, G. Hegenbart, E. Moisar, B. Pischel, and L. Wöste, Phys. Rev. Lett. 55, 3002 (1985).

${ }^{20}$ S. B. DiCenzo, S. D. Berry, and E. H. Hartford, Phys. Rev. B 38, 8465 (1988).

${ }^{21}$ W. Eberhardt, P. Fayet, D. M. Cox, Z. Fu, A. Kaldor, R. Sherwood, and D. Sondericker, Phys. Rev. Lett. 64, 780 (1990).

${ }^{22}$ W. Harbich, S. Fedrigo, F. Meyer, D. M. Lindsay, J. Lignieres, J. C. Rivoal, and D. Kreisle, J. Chem. Phys. 93, 8535 (1990).

${ }^{23}$ Z. Hu et al., J. Chem. Phys. 95, 2206 (1991); Z. Hu, B. Shen, J. R. Lombardi, and D. M. Lindsay, ibid. 96, 8757 (1992); S. Fedrigo, W. Harbich, and J. Buttet, Phys. Rev. B 47, 10706 (1993).

${ }^{24}$ Y. Kuk, M. F. Jarrold, P. J. Silverman, J. E. Bower, and W. L. Brown, Phys. Rev. B 39, 11168 (1989).

${ }^{25}$ J. E. Bower and M. F. Jarrold, J. Chem. Phys. 97, 8312 (1992).
${ }^{26}$ E. C. Honea, J. S. Kraus, J. E. Bower, and M. F. Jarrold, Z. Phys. D 26, 141 (1993).

${ }^{27}$ T. L. Haslett, K. A. Bosnick, and M. Moskovits, J. Chem. Phys. 108, 3453 (1998)

${ }^{28}$ Y. J. Chen, W. P. Chen, and E. Burstein, Phys. Rev. Lett. 36, 1207 (1976); K. Sakoda, K. Ohtaka, and E. Hanamura, Solid State Commun. 41, 393 (1982).

${ }^{29}$ S. Ushioda and Y. Sasaki, Phys. Rev. B 27, 1401 (1983); K. Kurosawa, R. M. Pierce, S. Ushioda, and J. C. Hemminger, ibid. 33, 789 (1986).

${ }^{30}$ E. C. Honea, A. Ogura, C. A. Murray, K. Raghavachari, W. O. Sprenger, M. F. Jarrold, and W. L. Brown, Nature (London) 366, 42 (1993).

${ }^{31}$ J. H. Weaver and G. D. Waddill, Science 251, 1444 (1991).

${ }^{32}$ T. Takagi, Z. Phys. D 3, 272 (1986); I. Yamada, C. J. Palmstron, E. Kennedy, J. W. Mayer, H. Inokawa, and T. Takagi, Mater. Res. Soc. Symp. Proc. 37, 401 (1985).

${ }^{33}$ M. F. Jarrold and J. E. Bower, J. Am. Chem. Soc. 111, 1988 (1979), and references therein.

${ }^{34}$ M. Moscovits, Rev. Mod. Phys. 57, 763 (1985).

${ }^{35} \mathrm{Al}_{2} \mathrm{O}_{3}$ Raman frequencies were taken from G. H. Watson, Jr., and W. B. Daniels, J. Appl. Phys. 52, 956 (1981).

${ }^{36}$ The peak value of the $\mathrm{N}_{2}$ Raman signal is plotted against angle in Fig. 5. The $1.2 \mathrm{~cm}^{-1}$ splitting of the Raman line, expected for the alpha phase of solid $\mathrm{N}_{2}$, was not resolved. See, R. Beck and J. W. Nibler, Chem. Phys. Lett. 159, 79 (1989).

${ }^{37}$ The optical constants used for the Ag film were taken from P. B. Johnson and R. W. Christy, Phys. Rev. B 6, 4370 (1972).

${ }^{38}$ M. D. Tillin and J. R. Sambles, J. Mod. Opt. 38, 731 (1991); W. Schlemminger and D. Stark, Thin Solid Films 137, 49 (1986).

${ }^{39}$ See, for instance, M. H. Brodsky in Light Scattering in Solids I, edited by M. Cardona (Springer-Verlag, Berlin, 1983), pp. 205-251, and references therein.

${ }^{40}$ R. G. Greenler and T. L. Slager, Spectrochim. Acta A 29, 193 (1973).

${ }^{41}$ M. Moskovits, J. Chem. Phys. 77, 4408 (1982).

${ }^{42}$ A. Campion in Vibrational Spectroscopy of Molecules on Surfaces, edited by J. T. Yates and T. E. Madey (Plenum, New York, 1987), pp. 360-373.

${ }^{43}$ The Raman intensities are related to the squares of the polarizability components: $\alpha_{x z}^{2}, \alpha_{y z}^{2}, \alpha_{y z} \alpha_{z z}$ and $\alpha_{z z}^{2}$. As discussed in the text, the largest contribution is from the $z$ oriented dipoles which are related to the $\alpha_{z z}^{2}$ term. In terms of the isotropic and anisotropic parts of the polarizability tensor, $\alpha_{z z}^{2}=\bar{\alpha}^{2}+(4 / 45) \gamma^{2}$. The $\alpha_{x z}^{2}$ and $\alpha_{y z}^{2}$ terms are $\alpha_{x z}^{2}=\alpha_{y z}^{2}$ $=(1 / 15) \gamma^{2}$. The $\alpha_{y z} \alpha_{z z}$ cross-term is zero.

${ }^{44}$ A. B. F. Duncan, in Chemical Applications of Spectroscopy, edited by W. West (Interscience, New York, 1956).

${ }^{45}$ The calculation was performed for a radiated wavelength of $496 \mathrm{~nm}$ (327 $\mathrm{cm}^{-1}$ vibrational frequency for $488 \mathrm{~nm}$ excitation), and a $\mathrm{N}_{2}$ matrix with $n_{1}=1.25$. The SPP field intensity was taken as $\sim \exp (-z / 700)(z$ in $\AA$ ) with a cluster/matrix thickness of $1400 \AA$. The optical constants of Johnson and Christy (Ref. 37) for Ag were used.

${ }^{46}$ For a description of the methods and basis sets, see W. J. Hehre, L. Radom, P. v. R. Schleyer, and J. A. Pople, Ab Initio Molecular Orbital Theory (Wiley, New York, 1986).

${ }^{47}$ A. D. McLean and G. S. Chandler, J. Chem. Phys. 72, 5639 (1980). The diffuse $s p$-exponent is 0.0331 ; the exponents for $d$ functions are 0.9 and 0.225 .

${ }^{48}$ S. Li, R. J. Van Zee, W. Weltner Jr., and K. Raghavachari, Chem. Phys. Lett. 243, 275 (1995).

${ }^{49}$ P. St. John and R. L. Whetten, Chem. Phys. Lett. 196, 330 (1992).

${ }^{50}$ Although the temperature was not continuously monitored during this time, the optical properties of the matrix were unchanged, indicating the temperature had not risen beyond $\sim 30 \mathrm{~K}$ where the matrix rapidly evaporates.

${ }^{51}$ M. H. Brodsky, in Light Scattering in Solids I, edited by M. Cardona (Springer-Verlag, Berlin, 1983), and references therein.

${ }^{52}$ D. Bermejo and M. Cardona, J. Non-Cryst. Solids 32, 405 (1979)

${ }^{53}$ J. C. Bourgoin, J. F. Morhange, and R. Beserman, Radiat. Eff. 22, 205 (1974); S. Roorda, W. C. Sinke, J. M. Poate, D. C. Jacobsen, S. Dierker, B. S. Dennis, D. J. Eaglesham, F. Spaepen, and P. Fuoss, Phys. Rev. B 44, 3702 (1991).

${ }^{54}$ Z. Iqbal, S. Veprek, A. P. Webb, and P. Capezzuto, Solid State Commun. 37, 993 (1981); Z. Iqbal and S. Veprek, J. Phys. C 15, 377 (1982).

${ }^{55}$ T. Okada, T. Iwaki, K. Yamamoto, H. Kasahara, and K. Abe, Solid State Commun. 49, 809 (1984); S. Hayashi and K. Yamamoto, Phase Transit. 24, 641 (1990), and references therein. 
${ }^{56}$ H. Richter, Z. P. Wang, and L. Ley, Solid State Commun. 39, 625 (1981); I. H. Campbell and P. M. Fauchet, ibid. 58, 739 (1986).

${ }^{57}$ S. Hayashi and K. Yamamoto, Supramol. Sci. 2, 581 (1986).

${ }^{58}$ T. Okada, H. Sasaki, S. Hayashi, S. S. Kim, and K. Yamamoto, Solid State Commun. 61, 671 (1987).
${ }^{59}$ J. Gonzalez-Hernandez and R. Tsu, Appl. Phys. Lett. 42, 90 (1983); J. S. Lannin, L. J. Pilione, S. T. Kshirsagar, R. Messier, and R. C. Ross, Phys. Rev. B 26, 3506 (1982); W. Sinke, T. Warabisako, M. Miyao, T. Tokuyama, S. Roorda, and F. W. Saris, J. Non-Cryst. Solids 99, 308 (1988). 